EPJ Web of Conferences 78, 06001 (2014)

DOI: $10.1051 /$ epjconf/20147806001

(C) Owned by the authors, published by EDP Sciences, 2014

\title{
Towards experiments at the new ELI-NP facility
}

\author{
D. L. Balabanski a , G. Cata-Danil, D. Filipescu, S. Gales, F. Negoita, O. Tesileanu, C. A. Ur, \\ I. Ursu, N. V. Zamfir, and the ELI-NP Science Team
}

ELI-NP, IFIN-HH, Reactorului Str. 30, 077125 Bucharest - Magurele, Romania

\begin{abstract}
The Extreme Light Infrastructure (ELI) Pan-European initiative represents a major step forward in quest for extreme electromagnetic fields. The Extreme Light Infrastructure - Nuclear Physics (ELI-NP) laboratory is one of the three pillars of the ELI project, that aims to use such extreme electromagnetic fields for nuclear physics and quantum electrodynamics research. At ELI-NP two ten petawatt high-power laser systems together with a very brilliant narrow-width $\gamma$ beam are the main research tools. Here the current status of the project and the experimental program related to nuclear research, which is under preparation at ELI-NP, are presented.
\end{abstract}

\section{The ELI-NP project}

ELI-NP [1] is one of the three laboratories under construction, related the ELI project [2], the other two being laser-driven secondary beams in Prague, the Czech Republic [3] and attosecond pulsed lasers in Szeged, Hungary [4]. In 2012 the 293-million-euro ELI-NP project was approved by the European Commission. ELI-NP will host two ultra high-power 10 PW lasers and a gamma beam system (GBS) which will deliver laser and $\gamma$-ray beams with parameters beyond those available at the present-stateof-the-art machines. The main laboratory building covers an area of more than $12000 \mathrm{~m}^{2}$. Its lay-out is displayed in Fig 1. The high-power laser system (HPLS) and the gamma beam system (GBS) are placed in the laboratory building, adjacent to the corresponding experimental areas. They will be mounted on an anti-vibration slab, damping vibrations to frequencies $\leq 10 \mathrm{~Hz}$ with amplitudes down to $\pm 1 \mu \mathrm{m}$.

The HPLS will have six output lines - two at $10 \mathrm{PW}$ with a frequency of $\geq 1 / 60 \mathrm{~Hz}$, two at $1 \mathrm{PW}$ with a frequency of $\geq 1 \mathrm{~Hz}$ and two at $100 \mathrm{TW}$ with a frequency of $\geq 10 \mathrm{~Hz}$. Each output will have its optical pulse compressor. The duration of the pulses from each of the six outputs of the HPLS shall be tunable from the best compression level to at least 5 ps pulse duration, with both positive and negative chirp. The HPLS outputs will be synchronized with accuracy below $200 \mathrm{fs}$ [5]. The laser system will deliver pulses synchronously with the GBS electron and $\gamma$ bunches.

The GBS is designed and constructed by the European consortium EuroGammaS, led by the Italian INFN LNF, including research and industrial partners from eight European countries. It will produce highly polarized ( $>95 \%$ ) tunable $\gamma$ beams of spectral density of $10^{4}$ photons/s/eV in the range from $200 \mathrm{keV}$ to $19.5 \mathrm{MeV}$ with a bandwidth of $>0.3 \%$ [6, 7]. The $\gamma$ beams will be produced through laser Compton backscattering (LCB) off an accelerated electron beam delivered by a linear

\footnotetext{
ae-mail: dimiter.balabanski@eli-np.ro
} 


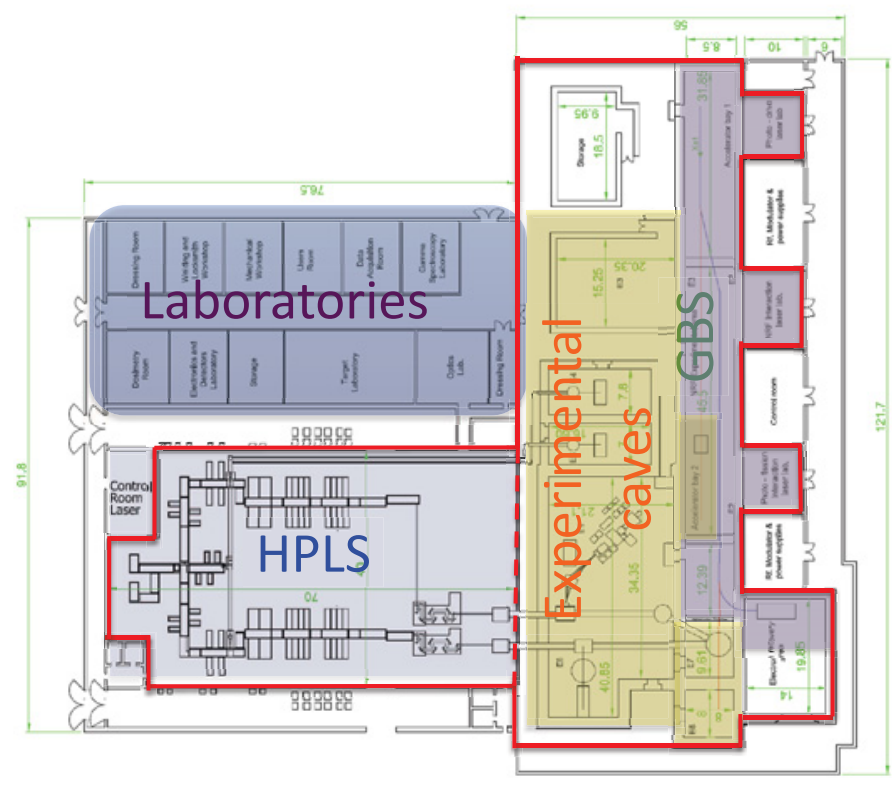

Figure 1. Layout of the ELI-NP laboratory building. The dimensions are in meters. The red contour indicates the borders of the anti-vibration slab.

accelerator. In the LCB process maximum up-shift is achieved in head-on collisions, producing $\gamma$ rays with energies $E_{\gamma} \sim 4 \gamma_{e}^{2} \cdot E_{L}$, where $\gamma_{e}=\left(1-\left(v_{e} / c\right)^{2}\right)^{-1 / 2}, v_{e}$ is the electron velocity and $E_{L}$ is the energy of the laser photons. For a green-light laser, $E_{L}=2.4 \mathrm{eV}$, LCB off $300-\mathrm{MeV}$ electrons results in a $\gamma$ beam of $E_{\gamma}<3.5 \mathrm{MeV}$, while LCB off $720-\mathrm{MeV}$ electrons yields $\gamma$ rays with $E_{\gamma}<20 \mathrm{MeV}$. The process has a relatively low cross section $\left(\sigma_{C B S} \approx 10^{-25} \mathrm{~cm}^{2}\right)$, which needs to be compensated by high photon and electron densities at the interaction point.

The solution for the electron accelerator is a a high-brightness normal-conducting electron Linac, combining S- and C-band accelerating cavities with the electrons provided by a photo-injector. The Linac produces high peak brightness in the single bunch, as well as a multi-bunch beam with effective high repetition rate, which increases the average current [8]. It will run at the maximum radiofrequency $(\mathrm{RF})$ repetition rate achievable $(100 \mathrm{~Hz})$, injecting from the photo-injectors into the booster Linac trains of bunches which are filling the available RF pulse time duration, typically, 32 bunches separated by 16 ns over a 512 ns flat RF pulse.

To optimize the $\gamma$-beam quality, an optical system preserving the spectral bandwidth while maximizing the flux must be provided at the Compton interaction point (IP). A passive optical system able to recirculate and focus several tens of time a single laser pulse at the Compton IP was designed [9]. The LINAC length and capabilities and the $\gamma$-ray beam characteristics impose strong constraints on the interaction laser. For the ELI-NP GBS the state-of-the-art high-power ytterbium laser technology [10] was adopted, e.g. laser beam wavelength $\lambda=515 \mathrm{~nm}$, and maximal laser pulse energy $U=400 \pm 5 \% \mathrm{~mJ}$ at the high-energy IP ( $U=200 \pm 5 \% \mathrm{~mJ}$ at the low-energy IP) with a pulse width $\sigma_{t}=5 \pm 5 \%$ ps and pulse repetition rate $100 \pm 5 \mathrm{~Hz}$. 
Wigner 111 - Colourful \& Deep Scientific Symposium
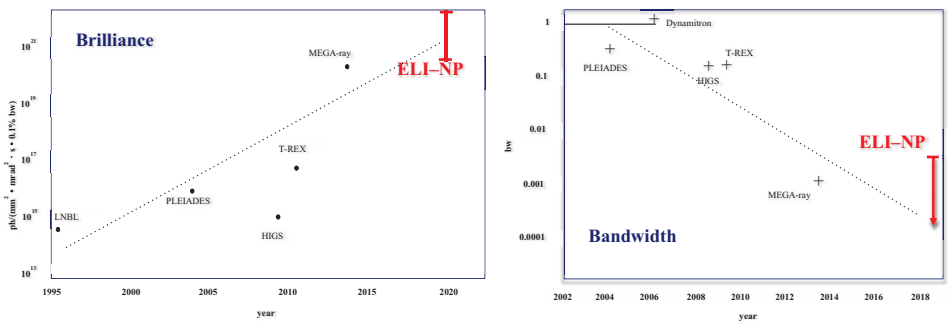

Figure 2. Comparison of the performance of the ELI-NP GBS with existing and planned CBS $\gamma$-beam facilities.

The ELI-NP GBS is superior to the existing or planned CBS $\gamma$-beam facilities in terms of brilliance and bandwidth, as demonstrated in Fig. 2. This becomes possible by taking advantage of several cutting-edge achievements in electron-beam and laser technologies, namely, by combining the delivery of high-intensity low-emittance electron beams with a very brilliant, high repetition-rate laser. The interaction takes place in a small volume, resulting in a brilliant narrow-width $\gamma$ beam.

\section{The ELI-NP Nuclear Science Programme}

The nuclear physics research program at ELI-NP consider both, experiments with ultra high-power lasers at the HPLS and experiments with brilliant $\gamma$ beams at the GBS. A large number of detectors and other instruments are being designed to achieve the goals of the ELI-NP experimental program [11].

\subsection{HPLS Nuclear Physics Experiments}

The HPLS, which was described in Sect. 1, will deliver every minute laser-light intensities on the target in the range of $10^{23} \mathrm{~W} / \mathrm{cm}^{2}$. This will allow to develop experiments using laser-driven heavyion nuclear reactions. For a recent review see Ref. [12]. Of special interest is to test the proposed fission-fusion reaction mechanism [13]. In this reaction, laser-accelerated actinide ions, e.g. ${ }^{232} \mathrm{Th}$, impinge on a ${ }^{232} \mathrm{Th}$ target, where the target-like and the beam-like Th ions will fission. Due to the very high beam intensity, exceeding existing classical ion beams by many (up to 15) orders of magnitude, high-temperature high-ion density will be created. As a result, subsequent fusion of neutron-rich fragments will occur, resulting in a very neutron-rich fusion product. The most important scientific objective in this case is the study of neutron-rich nuclei in the region of the r-process waiting point $N=126$, which can be achieved through fusion of two light neutron-rich fragments. Such experiments require huge experimental development in the field of laser driven ion acceleration.

\subsection{Nuclear Resonance Fluorescence Experiments with Narrow-Bandwidth $\gamma$-Ray Beams}

The advances in $\gamma$-ray beam brilliance and bandwidth and at ELI-NP, as discussed in Sect. 1 will increase the sensitivity of NRF experiments and thus it offers the opportunity to perform nuclear resonance fluorescence (NRF) studies on small target samples. This opens up an entire new area of applicability of the NRF method to materials that may be available only in quantities of a few milligrams. For example, studies the low-energy dipole response in the actinide region will be possible. The low-lying dipole strength, such as the scissors mode, and of the fragmentation of the strength 
of soft modes, such as the pigmy dipole resonance (PDR), will be studied, too. The brilliance of the $\gamma$-ray beam will enable $\gamma \gamma$ coincidence experiments, which provides an opportunity for detailed investigation of the decay of photo-excited states. Studies of the ground-state transition width $\Gamma_{0}$ with the self-absorption method [14] will benefit from the narrow bandwidth of the $\gamma$ beam. This will allow measurements of nuclear lifetimes in a model independent way with a very high precision. All these studies will benefit from the high polarization of the $\gamma$-ray beam.

\subsection{Photo-fission studies at the GBS}

The availability of a brilliant narrow-width $\gamma$-ray beam opens an avenue for photo-fission research, since it makes possible high-resolution studies in $\gamma$-ray induced reactions. This program will address high-resolution photo-fission experiments in the actinides, investigation of the second and third potential minima through studies of transmission resonances [15-17], angular and mass distribution measurements of fission fragments, measurements of absolute photo-fission cross-sections, studies of rare photo-fission events, such as ternary fission, highly asymmetric fission, etc. In addition, the possibility to produce exotic neutron-rich nuclei in photo-fission and study their structure and decays is investigated. The GBS $\gamma$-ray beam will cover selectively energy region of the giant dipole resonance (GDR) of the fissile target. The IGISOL technique [18] will be used for the extraction and selection of the isotopes of interest. The nuclei of interest will be slowed down and neutralized in the gas of an ion guide and will be separated by combining a laser-ion source and a mass separator. The isotope yield distribution was estimated, based in the measured fission yields in low-energy projectile fission of ${ }^{238} \mathrm{U}$ on ${ }^{208} \mathrm{~Pb}$ [19], a process which takes place via the exchange of a virtual photon $\left(E^{*}<25 \mathrm{MeV}\right)$. Estimates indicate that ELI-NP will compete other laboratories in studies of isotopes of refractory elements. In-beam $\gamma$-ray spectroscopy of fission fragments will be possible, too.

\subsection{GBS Experiments above the Neutron Threshold}

The brilliant, narrow-width, highly-polarized $\gamma$-ray beam which will be delivered by the ELI-NP GBS. will open up new horizons for the investigation of the nuclear photo-response at and above the separation threshold. An example for such studies is the detailed investigation of different soft modes, such as e.g. the PDRs at and above the particle threshold, which is essential for nucleosynthesis in astrophysics. PDRs are placed much lower in energy than the GDRs and they represent only a small fraction of the total E1 strength (few percent), while GDRs exhaust almost fully the E1 strength. Both, the GDR and the PDR can be covered within the energy range of the ELI-NP beams. The PDR occurs close to the neutron emission threshold and its decay is governed by the coupling to the large number of states around the threshold. These experiments will be complimentary to the PDR studies which were described in Sect. 2.2. This experimental program will include also studies of $(\gamma, \mathrm{n})$ cross section, e.g. p-process related measurements, such as the ${ }^{138} \mathrm{La}(\gamma, \mathrm{n}){ }^{137} \mathrm{La}$ and the ${ }^{180 m} \mathrm{Ta}(\gamma, \mathrm{n}){ }^{179} \mathrm{Ta}$ reactions, which are of key importance for the understanding of the process and provide constraints on the explosive dynamics of massive stars.

\subsection{GBS Nuclear Astrophysics Studies}

The principle of detailed balance allows the determination of the cross section of an $(\alpha, \gamma)$ process from the measurement of the time inverse $(\gamma, \alpha)$ reaction with $\gamma$-ray beams. Studying reactions by means of inverse photo-disintegration has the advantage of having different systematic uncertainties than those of charge-particle induced reactions measured at low energies of astrophysics interest. 
Wigner 111 - Colourful \& Deep Scientific Symposium

Several key nuclear astrophysics reactions were identified to be studied at ELI-NP. These include the ${ }^{12} \mathrm{C}(\alpha, \gamma){ }^{16} \mathrm{O}$ reaction at the Gamow peak $(300 \mathrm{keV})$, which is related to the carbon-to-oxygen ratio at the end of the He burning, one of the key open questions of nuclear astrophysics [20]. Other proposed studies include the ${ }^{24} \mathrm{Mg}(\gamma, \alpha)^{20} \mathrm{Ne}$ reaction, which governs the downward flow from ${ }^{24} \mathrm{Mg}$ to ${ }^{4} \mathrm{He}$. The ${ }^{22} \mathrm{Ne}(\gamma, \alpha){ }^{18} \mathrm{O}$ reaction, which is related to the production of ${ }^{22} \mathrm{Ne}$, which provides one of the neutron sources for s-process nucleosynthesis in massive stars through the ${ }^{22} \mathrm{Ne}(\alpha, \mathrm{n})$ reaction. The ${ }^{19} \mathrm{~F}(\gamma, \mathrm{p}){ }^{18} \mathrm{O}$ reaction, which is a measure for the loss of catalytic materials from the CNO cycle, providing at the same time a link to the $\mathrm{NeNa}$ cycle. The ${ }^{21} \mathrm{Ne}(\gamma, \alpha){ }^{17} \mathrm{O}$ reaction will allow the determination of the cross section at lower energies of the time-reversal ${ }^{17} \mathrm{O}(\alpha, \gamma)^{21} \mathrm{Ne}$ reaction, which is essential for determining the role of ${ }^{16} \mathrm{O}$ as a neutron poison in the $\mathrm{CNO}$ cycle.

\section{Conclusions}

The ELI-NP research center will host a HPLS and a GBS with parameters beyond nowadays stateof-the-art. The power of the HPLS lasers exceeds existing lasers by an order of magnitude and will deliver every minute on the target intensities in the range of $10^{23} \mathrm{~W} / \mathrm{cm}^{2}$. This makes it possible to design and perform new classes of nuclear physics experiments, which cannot be done elsewhere. The spectral density, brilliance and bandwidth of the $\gamma$-ray beams, which will be delivered by the GBS, are orders of magnitude better compared to the existing facilities. The outstanding performance of the $\gamma$ beam, combined with its high polarization, opens the possibility to carry out a versatile research program in nuclear physics and tackle key problems in nuclear structure, astrophysics and reactions.

\section{Acknowledgements}

The Extreme Light Infrastructure Nuclear Physics (ELI-NP) - Phase I project is co-funded by the European Union through the European Regional Development Fund.

\section{References}

[1] N. V. Zamfir, EPJ Web of Conferences 66, 11043 (2014)

[2] ELI - Extreme Light Infrastructure Science and Technology with Ultra-Intense Lasers Whitebook, eds. G A Mourou et al. (THOSS Media, Berlin, 2011)

[3] http://www.eli-beams.eu

[4] http://www.eli-hu.hu

[5] D. Ursescu et al., Proc. SPIE 8780, 87801H (2013)

[6] C. Vaccarezza et al., in Proc. IPAC 2012 Conference, TUOBB01 (2012)

[7] V. Petrillo et al., Nucl. Instr. Meth. A 693, 109 (2012)

[8] A. Bacci et al., J. Appl. Phys. 113, 194508 (2013)

[9] K. Dupraz et al., Phys. Rev. ST Accel. Beams 17, 033501 (2014)

[10] B. A. Reagan et al., Opt. Lett. 37, 3624 (2012)

[11] D. L. Balabanski et al., Acta Phys. Pol. B 45, 483 (2014)

[12] N. V. Zamfir, Eur. Phys. J. Special Topics 223, 1221 (2014)

[13] D. Habs et al., Appl. Phys. B 103, 471 (2011)

[14] N. Pietralla et al., Phys. Rev. C 51, 1021 (1995)

[15] P. G. Thirolf and D. Habs, Prog. Part. Nucl. Phys. 49, 352 (2002)

[16] A. Krasznahorkay, in Handbook of Nuclear Chemistry (Springer, Berlin, 2011) p. 281 
EPJ Web of Conferences

[17] L. Csige et al., Phys. Rev. C 87, 044321 (2013)

[18] J. Aysto et al., Phys. Rev. Lett. 69, 1167 (1992)

[19] C. Donzand et al., Eur. Phys. J. A 1, 407 (1998)

[20] W. A. Fowler, Rev. Mod. Phys. 56, 149 (1984) 